\title{
A group refactorization procedure for sleep electroencephalography
}

\author{
Robert G. Law and Shaun M. Purcell
}

December 29, 2020

Brigham and Women's Hospital, Boston MA

Harvard Medical School, Boston MA

\begin{abstract}
[WORKING DRAFT] In order to relate health and disease to brain state, patterns of activity in the brain must be phenotyped. In this regard, polysomnography datasets present both an opportunity and a challenge, as although sleep data are extensive and multidimensional, features of the sleep EEG are known to correlate with clinical outcomes. Machine learning methods for rank reduction are attractive means for bringing the phenotyping problem to a manageable size. The whole-night power spectrogram is nonnegative, and so applying nonnegative matrix factorization (NMF) to separate spectrograms into time and frequency factors is a natural choice for dimension reduction. However, NMF converges differently depending on initial conditions, and there is no guarantee that factors obtained from one individual will be comparable with those from another, hampering inter-individual analysis.

We therefore reseed time-frequency NMF with group frequency factors obtained from the entire sample. This "refactorization" extends classical frequency bands to frequency factors. The group reseeding procedure coerces factors into equivalence classes, making them comparable across individuals. By comparing frequency factor properties, we illustrate age-related effects on the sleep EEG. The procedure can presumably be adapted to higher resolutions, e.g. to local field potential datasets, for characterizing individual time-frequency events.
\end{abstract}

\section{Introduction}

With the advent of large-scale neural time-series datasets, an open question is how best to distill these data into quantitative phenotypes. Nowhere is this more pertinent than in polysomnography, where a single recording session includes upwards of eight hours of electroencephalography (EEG) as well as a variety of other physiological time series. A common reduction of data in sleep EEG is 
to calculate the power in a small set of frequency bands for each epoch, and then examine the dynamics of bandlimited power over the course of the night [2]. Power in each of these bands typically corresponds to oscillatory intensity, number, and/or extent and are markers for a variety of healthy and diseased states (e.g. $[1,3,20,13])$. Yet it is well-known that the frequency modes of comparable oscillations vary across individuals and with age (e.g. [3]), and despite nearly a century of research there remains disagreement on even the boundaries of each frequency band (compare, for instance, [15] to [7]).

In small datasets such definitional issues can be dealt with heuristically, but in large datasets they may be more problematic: Directly comparing $20 \mathrm{~Hz}$ activity across a population is suboptimal, if $20 \mathrm{~Hz}$ activity in one individual is functionally equivalent to $18 \mathrm{~Hz}$ activity in another. Furthermore, the observed activity at a specific frequency bin (e.g. $15 \mathrm{~Hz}$ ) or band (e.g. sigma) may reflect a summation across a heterogeneous mixture of components, which may individually exhibit qualitatively distinct patterns of association, or dynamics, whereas analyses based only on the observed spectrogram may obscure such features.

Our contribution here is to establish a data reduction procedure based on nonnegative matrix factorization (NMF; [14]) that constructs individualized frequency factors that can be meaningfully interpreted and compared at the population level, and which potentially deconvolves distinct but overlapping spectral signatures, and to show that these factors vary with age in an interpretable way.

\section{Methods}

\subsection{Datasets}

We studied two datasets from the National Sleep Research Resource polysomnography database [22]. The Childhood Adenotonsillectomy Trial (ChAT) was chosen for future examination of test/retest correlations ([17]; $n=859$ recording sessions; 452 baseline, 407 followup; aged 5-10 years), while the Cleveland Family Study (CFS) was chosen as a dataset for investigating age correlations here ([18]; $n=540$ individuals; aged $7-89$ years; see below) .

\subsection{Preprocessing}

All preprocessing was performed using Luna, a freely available command-line utility specialized for handling large-scale sleep data (http://zzz . bwh . harvard. edu/luna). Each EEG time-series (channel C3; contralateral mastoid reference) was converted to microvolts and partitioned into nonoverlapping, contiguous 30 -second epochs. A spectrum for each epoch was estimated using the Welch method $(0.5-45 \mathrm{~Hz} ; 0.5 \mathrm{~Hz}$ bins $)$. For each time-series with $m$ epochs, this yields a non-overlapping spectrogram $\mathbf{Y}$, a $90 \times m$ matrix representing the time-frequency plane. Further analysis was restricted to sleep and brief arousals within sleep cycles [6]. 
For polysomnograms in the Cleveland Family Study, we removed all sessions where sleep onset time was before 10pm, as participants were awoken at this time for clinical assessment. Due mainly to the denoising nature of NMF [21], we found that no further artifact rejection was necessary for our purposes here.

\subsection{Factorization}

Nonnegative matrix factorization [14] finds a fixed-rank approximation $\mathbf{W H} \approx$ $\mathbf{Y}$ with all entries nonnegative, enabling a "parts-based" decomposition [10] in the sense that each epoch of spectral data is reconstructed by combining a small number of spectral ingredients w ("what") in various amounts $\mathbf{h}$ ("how much"). This corresponds to the notion that a spectrum is generated by a finite collection of processes, each with its own spectrum, that are combined at the scalp-level. Unlike global methods like principal components analysis, the approximation reached by NMF depends critically on the initial seed matrices $\mathbf{W}_{0}, \mathbf{H}_{0}$. While many applications of nonnegative matrix factorization use random seeds [4], "warm-start" factorization methods [5] can outperform random seeds, although they rely on the approximate correctness of an initial guess.

Here, we construct such an initial guess by sampling from the population. Spectrograms were first $\log$-transformed and minimized $\mathbf{Y}^{\prime} \leftarrow \log (\mathbf{Y} / \min \mathbf{Y})$, so that $\min \mathbf{Y}^{\prime}=0$. All factorizations were then performed using the multiplicative method [10] implemented in the NMF R package [8]; see Figure 1 for an example.

We take a three-step approach (Figure 2): We first presuppose a nearlyuniform seed, where $\mathbf{H}_{\mathbf{0}}$ is uniform $\left(h_{i j}=1\right)$ and $\mathbf{W}_{\mathbf{0}}$ differs from uniform only enough to break symmetry in the initial conditions $\left(w_{i, i}=2 ; w_{i j}=1\right.$ otherwise). This yields initial factorizations for each individual $k: \mathbf{W}_{k}, \mathbf{H}_{k}$. We discard the temporal factors $\mathbf{H}$, and then concatenate the frequency factors from this first factorization, as $\mathbf{W}_{1}\left|\mathbf{W}_{2}\right| \cdots\left|\mathbf{W}_{k}\right| \cdots \mid \mathbf{W}_{n}$. We factorize this concatenated matrix to obtain six group-level frequency factors in a $90 \times 6$ matrix $\boldsymbol{\Omega}$. These are used as frequency seeds (again with uniform temporal seeds $\mathbf{H}_{0}$ ) for a second NMF round on individuals, from which the final frequency and temporal factors are obtained.

More informally, we should like to: 1) use NMF with a nearly-uniform seed to find six spectral ingredients in each individual's sleep 2) Put all $6 \times n$ individual ingredients together and use NMF to find six "base ingredients", and then 3) Look for these base ingredients (and variations thereof) by seeding a second NMF round with the base ingredients. We examine time and frequency factors obtained from this second round of factorization.

Note that when we simply used the factors obtained from (1), the factorization was not stable: i.e. similar factors were permuted across sessions, making comparisons of factors across sessions effectively impossible. Evidently, though, the group seed $\boldsymbol{\Omega}$ contains zero entries unique for each factor (Figure 2B), all of which are preserved by the NMF update rule. As such, each factor has a unique support that is preserved in each iteration of NMF: This ultimately defines an equivalence class of probability density functions (e.g. normalized spectra) 
bioRxiv preprint doi: https://doi.org/10.1101/2020.12.29.424768; this version posted December 30,2020. The copyright holder for this preprint (which was not certified by peer review) is the author/funder, who has granted bioRxiv a license to display the preprint in perpetuity. It is made available under aCC-BY-NC 4.0 International license.

A


B

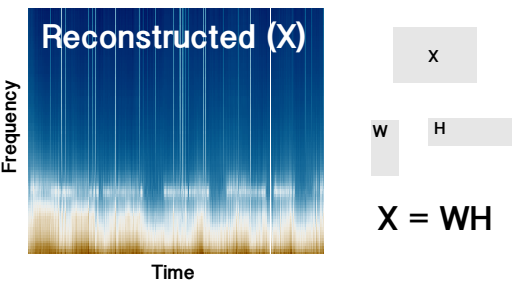

Figure 1: A) Factorization of a log-spectrogram $\mathbf{Y}$ into nonnegative frequencyand time-components $\mathbf{W}$ and $\mathbf{H}$. The initial conditions for each factorization are shown as arrows. B) Approximate spectrogram, reconstructed by multiplication of the factors. 


\section{A}

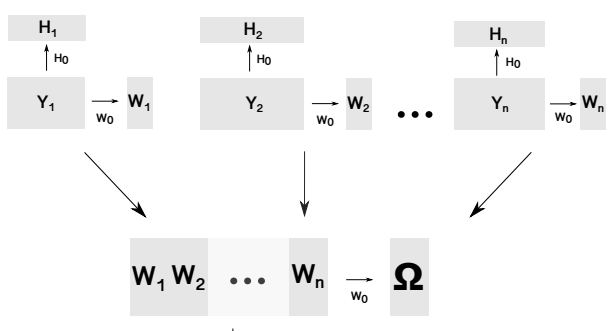

$\boldsymbol{0}$

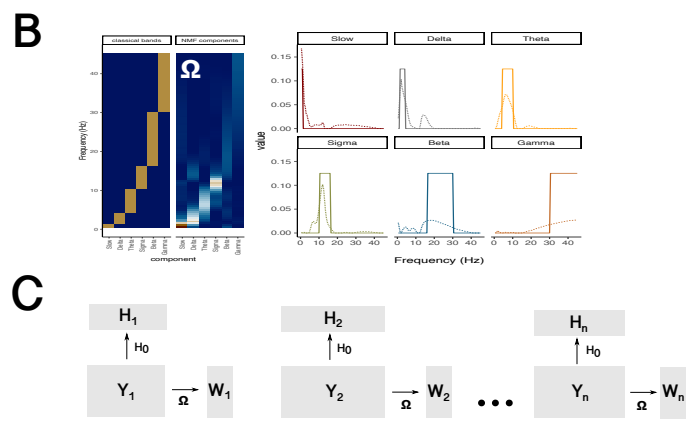

Figure 2: A) The $\mathbf{W}_{k}$ obtained from a first factorization using nearly-uniform seeds $\mathbf{W}_{0}, \mathbf{H}_{0}$ (see text) are concatenated and themselves factored to obtain a set of group factors $\boldsymbol{\Omega}$. B) Comparison of classical frequency bands to $\Omega$-factors as heatmaps (left) and as functions (right) $\mathbf{C}) \boldsymbol{\Omega}$-factors are then used as seeds to obtain a final factorization. 
admissible for each factor, which is what makes within-class comparisons reasonable.

\section{Results}

\subsection{NMF frequency factors extend classical frequency bands}

Inspection of the frequency factors then yielded an immediate visual match to classical frequency bands in both the ChAT and CFS datasets (Figure 3A/B) , although the classical theta and alpha bands merged into one factor (denoted "theta" henceforth). The primary difference between classical bands and NMF factors is again one of support: the w-factors - while concentrated within classical bandlimits - are also supported outside those limits. Individual $\mathbf{w}$-factors clustered near their respective means. Factors were comparable between the two datasets (Figure 3C), but all aside from the "gamma" factor were distinguishable between ChAT and CFS, with presumably age-dependent shifts evident in "delta", "theta", "sigma", and "beta" factors.

\subsection{Frequency factors reveal age-dependent cross-frequency coupling}

We next considered how frequency factors were correlated with age - a wellstudied demographic covariate of sleep dynamics (Figure 4). We found that age differences within factors tended to occur when spectral power can be grouped preferentially into either one factor or another at the population level. For instance, since the "delta" factor and the "sigma" factor have support near $15 \mathrm{~Hz}$, at each epoch NMF can couple each unit of $15 \mathrm{~Hz}$ power to either $1-4 \mathrm{~Hz}$ or $\sim 12 \mathrm{~Hz}$ power. We find a strong age-dependence to this assignment, with aging associated with less assignment of $15 \mathrm{~Hz}$ power to the "delta" factor, reflecting reduced coupling of $15 \mathrm{~Hz}$ power to the $1-4 \mathrm{~Hz}$ delta mode with age (Figure $4 \mathrm{~A}$ ). With increasing age, $15 \mathrm{~Hz}$ power is instead assigned to the "sigma" factor (Figure $4 \mathrm{~B})$. In other words, fast spindles appear to decouple from slow oscillations with age, agreeing with results from a recent slow-wave stimulation experiment [19]. Coupling of $10 \mathrm{~Hz}$ power to either the "theta" or "slow" factor was also agedependent (Figure 4C/D).

\section{Discussion}

We have developed a procedure for extracting comparable EEG phenotypes in sleep using nonnegative matrix factorization, showing that group refactorization successfully decomposes power spectra into frequency factors resembling but extending classical frequency bands, with age-dependent features. While our efforts here were primarily concerned with validating the procedure, applications may range from cross-species comparisons of factors to subepoch characteriza- 

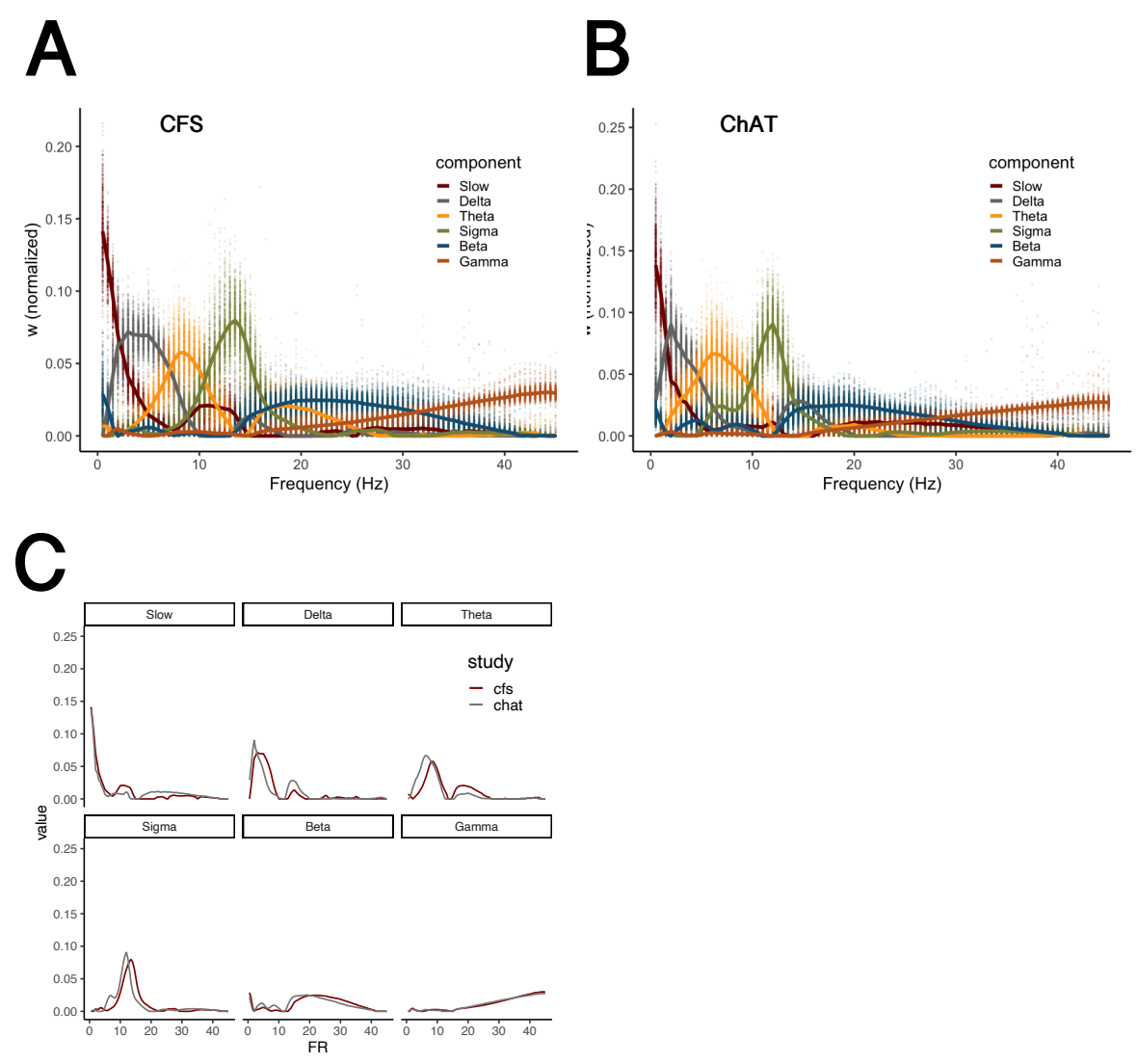

Figure 3: Frequency factors in the Childhood Adenotonsillectomy Trial and Cleveland Family Study. A) ChAT frequency factors. Mean and all individual data points are shown. Inset: Strength of traitlikeness is indicated by the zscore of the actual test/retest correlation relative to a distribution of correlations under shuffled session IDs. B) CFS fyequency factors. C) Left: example of frequency factors with good test/retest correlation. Right: example with poor correlation. D) Overlaid mean frequency factors from the ChAT and CFS datasets. 

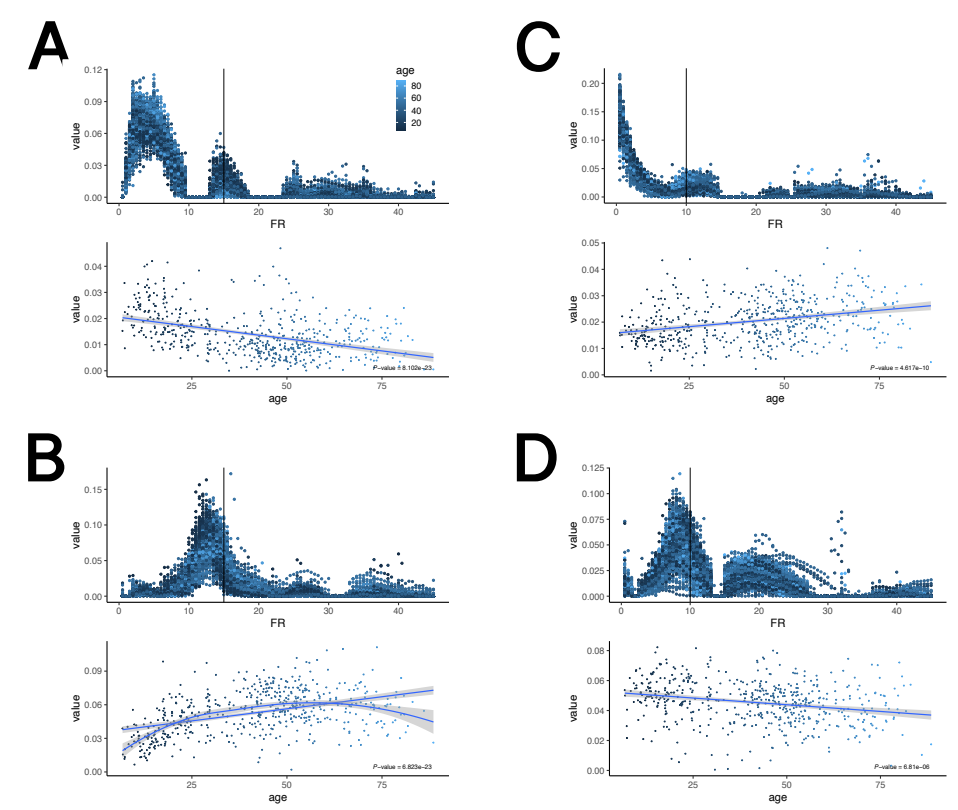

D
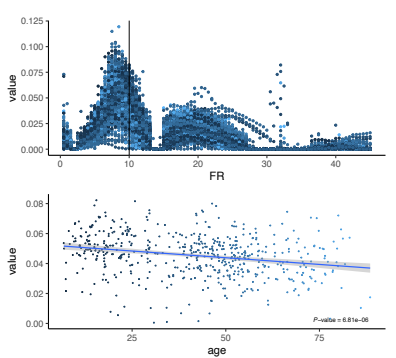

Figure 4: Frequency factor correlates with age. A) Top: Individual "Delta" frequency factors are plotted, colored by age. The vertical line represents a slice through the frequency of interest, revealing (bottom) an age-anticorrelated $15 \mathrm{~Hz}$ component. B) "Sigma" factor has a corresponding age-correlated $15 \mathrm{~Hz}$ component. C) "Slow" factor has an age-correlated $10 \mathrm{~Hz}$ component. D) "Theta" factor has a corresponding age-anticorrelated $10 \mathrm{~Hz}$ component. All p-values are uncorrected (540 comparisons). 
tion of individual time-frequency events such as slow waves and sleep spindles, along the lines of [16].

Nonnegative methods have been applied to polysomnography previously: The most similar approach to ours is [9], who used nonnegative tensor factorization with twelve fixed frequency bands to discriminate spectrotemporal patterns in Alzheimer's disease, but this factorization was supervised. Tensor factorization, moreover, cannot be applied to sleep recordings with variable duration without truncation; this indeed was a primary motivation in developing our method. Other related approaches are [11] and [12]: [11], in particular, applied kernel NMF to obtain frequency factors within individuals; these factors, however, were not compared across individuals as the primary motivation for the study was brain-computer interface design.

It is interesting that we were able to obtain our results with a standard NMF algorithm using only manipulations of the seed matrices: It is not completely clear why the uniform seed $\mathbf{H}_{0}$ appears to yield sufficient smoothness for our purposes, nor why the group seed $\boldsymbol{\Omega}$ is sparse enough to assure equivalance of factors across individuals. However, given these findings, we are left with the possibility that in combination with experiment and modeling, phenotypic factors obtained from these epidemiological-scale datasets might be mapped to physiological properties of cells or networks, in a more direct manner than using classical frequency bands.

\section{References}

[1] P. Achermann and A. A. Borb $\widetilde{A}($ ly. Temporal evolution of coherence and power in the human sleep electroencephalogram. Journal of Sleep Research, 7 Suppl 1:36-41, 1998.

[2] Daniel Aeschbach and Alexander A. BorbÃ@(ly. All-night dynamics of the human sleep EEG. Journal of Sleep Research, 2(2):70-81, 1993. _eprint: https://onlinelibrary.wiley.com/doi/pdf/10.1111/j.13652869.1993.tb00065.x.

[3] Roy Cox, Anna C. Schapiro, Dara S. Manoach, and Robert Stickgold. Individual Differences in Frequency and Topography of Slow and Fast Sleep Spindles. Frontiers in Human Neuroscience, 11, 2017. Publisher: Frontiers.

[4] Karthik Devarajan. Nonnegative Matrix Factorization: An Analytical and Interpretive Tool in Computational Biology. PLOS Computational Biology, 4(7):e1000029, July 2008. Publisher: Public Library of Science.

[5] Marco Enriquez. Classification of Air Traffic Controller Utterance Transcripts via Warm-Start Non-Negative Matrix Factorization. Air Traffic Control Quarterly, 22(2):137-156, 2014. Publisher: American Institute of Aeronautics and Astronautics _eprint: https://doi.org/10.2514/atcq.22.2.137. 
[6] I. Feinberg. Changes in sleep cycle patterns with age. Journal of Psychiatric Research, 10(3-4):283-306, October 1974.

[7] R. Ferri, F. I. Cosentino, M. Elia, S. A. Musumeci, R. Marinig, and P. Bergonzi. Relationship between Delta, Sigma, Beta, and Gamma EEG bands at REM sleep onset and REM sleep end. Clinical Neurophysiology: Official Journal of the International Federation of Clinical Neurophysiology, 112(11):2046-2052, November 2001.

[8] Renaud Gaujoux and Cathal Seoighe. A flexible R package for nonnegative matrix factorization. BMC Bioinformatics, 11(1):367, July 2010.

[9] Yejin Kim, Xiaoqian Jiang, Luyao Chen, Xiaojin Li, and Licong Cui. Discriminative Sleep Patterns of Alzheimer's Disease via Tensor Factorization. arXiv:1905.05827 [cs, q-bio], May 2019. arXiv: 1905.05827.

[10] Daniel D. Lee and H. Sebastian Seung. Learning the parts of objects by nonnegative matrix factorization. Nature, 401(6755):788-791, October 1999. Number: 6755 Publisher: Nature Publishing Group.

[11] Hyekyoung Lee, Andrzej Cichocki, and Seungjin Choi. Kernel nonnegative matrix factorization for spectral EEG feature extraction. Neurocomputing, 72(13-15):3182-3190, August 2009.

[12] Sheng Liu, Mark Cheng, Hayley Brooks, Wayne Mackey, David J. Heeger, Esteban G. Tabak, and Carlos Fernandez-Granda. Time-Series Analysis via Low-Rank Matrix Factorization Applied to Infant-Sleep Data. arXiv:1904.04780 [cs, stat], November 2019. arXiv: 1904.04780.

[13] Dara S. Manoach, Jen Q. Pan, Shaun M. Purcell, and Robert Stickgold. Reduced sleep spindles in schizophrenia: A treatable endophenotype that links risk genes to impaired cognition? Biological psychiatry, 80(8):599608, October 2016.

[14] Pentti Paatero and Unto Tapper. Positive matrix factorization: A non-negative factor model with optimal utilization of error estimates of data values. Environmetrics, 5(2):111-126, 1994. _eprint: https://onlinelibrary.wiley.com/doi/pdf/10.1002/env.3170050203.

[15] Michael L. Perlis, Helli Merica, Michael T. Smith, and Donna E. Giles. Beta EEG activity and insomnia. Sleep Medicine Reviews, 5(5):365-376, October 2001.

[16] Michael J. Prerau, Ritchie E. Brown, Matt T. Bianchi, Jeffrey M. Ellenbogen, and Patrick L. Purdon. Sleep Neurophysiological Dynamics Through the Lens of Multitaper Spectral Analysis. Physiology (Bethesda, Md.), 32(1):60-92, January 2017. 
[17] Susan Redline, Raouf Amin, Dean Beebe, Ronald D. Chervin, Susan L. Garetz, Bruno Giordani, Carole L. Marcus, Renee H. Moore, Carol L. Rosen, Raanan Arens, David Gozal, Eliot S. Katz, Ronald B. Mitchell, Hiren Muzumdar, H.G. Taylor, Nina Thomas, and Susan Ellenberg. The Childhood Adenotonsillectomy Trial (CHAT): Rationale, Design, and Challenges of a Randomized Controlled Trial Evaluating a Standard Surgical Procedure in a Pediatric Population. Sleep, 34(11):1509-1517, November 2011.

[18] Susan Redline, Peter V. Tishler, Tor D. Tosteson, John Williamson, Kenneth Kump, Ilene Browner, Veronica Ferrette, and Patrick Krejci. The Familial Aggregation of Obstructive Sleep Apnea. American Journal of Respiratory and Critical Care Medicine, 151(3_pt_1):682-687, March 1995. Publisher: American Thoracic Society - AJRCCM.

[19] Jules Schneider, Penelope A Lewis, Dominik Koester, Jan Born, and HongViet V Ngo. Susceptibility to auditory closed-loop stimulation of sleep slow oscillations changes with age. Sleep, 43(zsaa111), December 2020.

[20] Hyeyoung Shin, Robert Law, Shawn Tsutsui, Christopher I Moore, and Stephanie R Jones. The rate of transient beta frequency events predicts behavior across tasks and species. eLife, 6:e29086, November 2017. Publisher: eLife Sciences Publications, Ltd.

[21] Kevin W. Wilson, Bhiksha Raj, Paris Smaragdis, and Ajay Divakaran. Speech denoising using nonnegative matrix factorization with priors. In 2008 IEEE International Conference on Acoustics, Speech and Signal Processing, pages 4029-4032, Las Vegas, NV, USA, March 2008. IEEE. ISSN: 1520-6149.

[22] Guo-Qiang Zhang, Licong Cui, Remo Mueller, Shiqiang Tao, Matthew Kim, Michael Rueschman, Sara Mariani, Daniel Mobley, and Susan Redline. The National Sleep Research Resource: towards a sleep data commons. Journal of the American Medical Informatics Association: JAMIA, 25(10):1351-1358, October 2018. 